\title{
Frequency and Voltage Partitioning in Presence of Renewable Energy Resources for Power System(Example: North Chile Power Network)
}

\author{
Hasan Mehrjerdi \\ Nasser A. Al-Emadi \\ Electrical Engineering Department \\ Qatar University \\ Doha, Qatar
}

\author{
Elyad Rakhshani \\ Pedro Rodriguez \\ Electrical Engineering Department \\ Technical University of Catalonia \\ Barcelona, Spain
}

\begin{abstract}
This paper investigates techniques for frequency and voltage partitioning of power network based on the graph-theory. These methods divide the power system into distinguished regions to avoid the spread of disturbances and to minimize the interaction between these regions for frequency and voltage control of power system. In case of required active and reactive power for improving the performance of the power system, control can be performed regionally instead of a centralized controller. In this paper, renewable energy sources are connected to the power network to verify the effect of these sources on the power systems partitioning and performance. The number of regions is found based on the frequency sensitivity for frequency partitioning and bus voltage for voltage partitioning to disturbances being applied to loads in each region. The methodology is applied to the north part of Chile power network. The results show the performance and ability of graph frequency and voltage partitioning algorithm to divide large scale power systems to smaller regions for applying decentralized controllers.
\end{abstract}

Index Terms - Chile Power Network, Graph Theory, Secondary Frequency Partitioning, Voltage Partitioning.

\section{INTRODUCTION}

T oday's power systems are encountering a fundamental reinfrastructure considering the renewable energy resources (RES) connecting to their structure. RES can affect frequency and voltage in power systems. High penetration of renewable resources and required technology for integration of these new resources provide new challenges for researches. Therefore, the recent trends of researches are through the adoption of previous concepts and conventional models with high penetration of RES [1]-[3].

The way of controlling the frequency and voltage of power systems are different. Centralized control become complex and insufficient. Decentralized control for frequency and voltage can be as a replacement techniques. Decentralized control is a technique that can be adequate and effective for large scale power systems using local controllers. The first step of decentralized control for a large scale power system is partitioning. Here, two different methods are presented to partition large scale power systems to smaller regions using the Graph theory algorithm. It is shown that the results of partitioning for frequency and voltage are different. It means different partitions are obtained for frequency and voltage control. Distances between buses and power flow results play an important role into the frequency and voltage partitioning of the power systems. With increasing of RES in power systems, it is vital to consider the effect of RES integration into partitioning of the power systems. Here, for a case study, North Chile Power Network (NCPN) is considered. The RES including PV and Thermal Solar have been connected to the ATACAMA region. The partitioning can elaborate the idea of secondary frequency and voltage control as the power industry is investing to protect power system networks against different disturbances, but large interconnected power systems require innovative solutions to make these practical. Although the literature presents several studies on the partitioning problem, but the unique power network of Chile have specific challenges. In Chile, transmission systems have long lines with loads remote from production. There are a lot of mines connected to the network that can affect quality of the network and its performance. It is generally difficult to maintain constant frequency and voltage when the demand varies under highly loaded conditions. Without control, the frequency and voltage will exceed its operating range and is harmful to the quality and security.

This motivates us to study and find efficient and secure frequency and voltage partitions for power systems by future controls suitable to real-time and large networks. The goal is to maintain frequency and voltage within the ranges permitted.

Different methods have been developed for partitioning recognition and model reduction [4]-[12].

The graph partitioning is open area of research and involving many different branches of engineering. The use of graph representation is employed to simplify the structure of power systems and utilizes the following systems: branches 
representing transmission lines and transformers, nodes representing buses in the power systems, weights of a branch equal to the active/reactive power flow in MW/MVAR through the line or a transformer representing the branch [13]-[17].

This paper describes a spectral graph-based partitioning method to perform a k-way partitioning for frequency and voltage based on the k-dimensional spectral embedding of a graph [18]. In spectral partitioning techniques, the eigenvectors and eigenvalues of a graph are computed and a cost function is shown to be minimized by a function of the spectrum. The proposed scheme separates the large scale power system into smaller partitions to avoid cascading events.

The scheme proposed in this paper separates the power system into smaller regions based on frequency and voltage using graph theory with the goal to avoid cascading events. The algorithm is tested on the NCPN network. This network is divided into regions that are designed to have minimum interaction and therefore any disturbances in a particular region will not strongly propagate to the other regions. The regions can be reconfigured and updated in accordance with variations in grid structure. In this paper, disturbances are defined as increasing of active and reactive power into the loads.

In this paper different weights have been considered for the frequency and voltage partitioning accordingly. Furthermore, RES have been connected to the power network and partitioning have been performed to see the effect of the RES on the partitioning of the power network.

The paper is organized as follows: Section II presents the frequency partitioning algorithm based on the graph theory. Section III describes frequency partitioning considering the RES connected to the power system. Section IV demonstrates the voltage partitioning algorithm. Section V presents the voltage partitioning algorithm with connection of RES. Section VI presents the conclusion.

\section{FREQUENCY PARTITIONING AlgORITHM}

For frequency control, as figure 1 shows, frequency and variation of frequency of each partition will be measured and active power will be injected into partitions to compensate frequency variations. The importance of frequency partitioning is to obtain the active power transferred between buses on transmission lines in different contingencies. A power plow in different situations considering different contingencies will be considered to verify the partitions.

The NCPN has eight generators, which one of them is in Argentina and will not be considered in our study. A PV and Thermal Solar plants are connected to the area of ATACAMA and will penetrate about $200 \mathrm{MW}$ to the network.

A power flow has been performed in normal situation of the network to find the active and reactive power flowing on the lines connected between buses. This information will be used as weighting of graph connection matrix between buses.

Active power will be used for frequency partitioning technique. The weights on the transmission lines for the frequency partitioning are calculated as follows:

\section{A.1 Weighted Graph model of the NCPN}

$$
\omega_{i j}=P_{i j}
$$

where $P_{i j}$ is the active power transferred on the transmission lines between buses.

The frequency graph partitioning problem involves a graph, $G=(\mathcal{V}, \mathcal{E})$ with vertices, $B=\left\{b_{1}, b_{2}, \ldots, b_{n}\right\}$ and weighted edges where the weight of edge $P_{i j}=\left(b_{i}, b_{j}\right)$ represents the active power transferred between $b_{i}$ and $b_{j}$.

We associate to $\mathrm{G}$ a weight matrix $\omega$ that satisfies the following properties:

$$
\begin{aligned}
& \text { (1) } \omega_{\mathrm{ij}}=\omega_{\mathrm{ji}} \\
& \text { (2) } \omega_{\mathrm{ii}}=0 .
\end{aligned}
$$

(3) $\omega_{\mathrm{ij}} \geq 0$ and $\omega_{\mathrm{ij}}=0$ if $\mathrm{i}$ is not adjacent to $\mathrm{j}$ in $\mathrm{G}$.

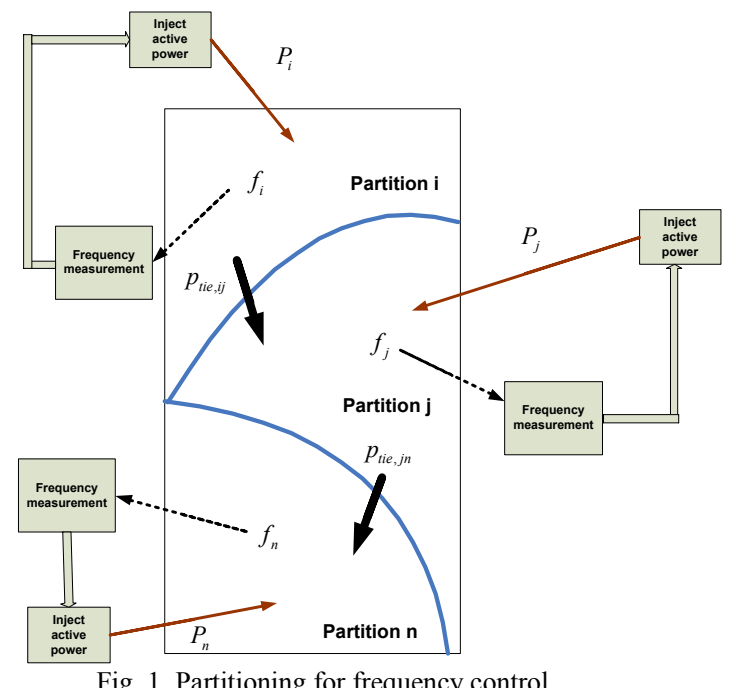

The problem is to find a partition for the set of vertices $\mathcal{P}=$ $\left\{p_{1}, p_{2}, \ldots, p_{k}\right\}$ for a given $k$ that optimizes some cost criterion based on the weights of edges. Spectral-based partitioning extracts global information about the structure of a graph from eigenvalues/eigenvectors of graph matrices.

The relation between the properties of a graph and its spectrum are studied in [22]. The adjacency matrix of $G$ is the $n \times n$ matrix $\mathrm{A}(\mathrm{G})=\left[a_{\mathrm{ij}}\right]$ where $\mathrm{a}_{\mathrm{ij}}$ is the weight of the edge between $b_{i}$ and $b_{j}$. The degree matrix of $\mathrm{G}$ is the $\mathrm{n} \times \mathrm{n}$ matrix $\mathrm{D}(\mathrm{G})=\left[\mathrm{d}_{\mathrm{ij}}\right]$ is defined by

$$
d_{i j}=\left\{\begin{array}{cl}
\sum_{k=1}^{n} a_{i k} & \text { if } i=j \\
0 & \text { if } i \neq j
\end{array}\right.
$$

The Laplacian of $G$ is the $n \times n$ symmetric matrix $L(G)=$ $D(G)-A(G)$. Since the rows (and columns) sum to 0 , the Laplacian is singular, it has rank at most $n-1$ and it has 0 as eigenvalue. The matrix $L(G)$ is positive semidefinite and has only real eigenvalues. Thus the smallest eigenvalue is 0 and all other eigenvalues are positive. The multiplicity of 0 as an eigenvalue represents the number of connected sub-graphs. 
$\lambda_{1}, \lambda_{2}, \ldots, \lambda_{k}$ are the $k$ smallest eigenvalues of the matrix $L$ and $x_{1}, x_{2}, \ldots, x_{k}$ are the eigenvectors associated with eigenvalues from which the first eigenvectors partition vector will be built. The partition matrix $\hat{C}$ is defined as:

$$
\hat{C}=Z(X) X X^{T} Z(X)
$$

where $Z(X)$ is a diagonal matrix with

$$
z_{i j}=\frac{1}{\sqrt{\sum_{h=1}^{k} x_{i h}^{\prime 2}}}
$$

$\hat{C}_{i j}$ is the cosine of the angle between the two row vectors $i$ and $j$ and represent the closeness of vertices to each other. The first vertex is chosen at random and can be considered as center of the first region. To find the second vertex, or the center of the second partition, $\hat{C}_{i c_{1}}$ for $i=1, \ldots, n$ is calculated to find the minimum of $\hat{C}_{i c_{1}}$, therefore the second center of the region is found. During searching other regions to find the $k$ region, the following equation is used:

$$
\operatorname{Min} \mathrm{Y}=\left(1-\hat{C}_{i c_{1}}\right)+\left(1-\hat{C}_{i c_{2}}\right)+\cdots+\left(1-\hat{C}_{i c_{k}}\right)
$$

\section{A. $2 \quad N C P N$ frequency graph partitioning}

Graph theory algorithm for frequency partitioning can be considered as follow:

- Numbering the buses (Loads and Generations)

- Finding connection matrix

- Weighting connection matrix

- Finding Degree matrix

- Finding Laplacian matrix

- Finding eigenvalue and eigenvectors

- Finding partitions

The Graph Theory algorithm has been performed to find three partitions for NCPN. The results can be seen in the figure 2 . As can be seen in this figure, firstly, three partitions have been considered.

In this partitioning, PV and Thermal Solar are not connected to the NCPN. Buses are including in each partition has been differently colored. The active power transferred on the transmission lines are considered as weights and partitioning has been performed based on power flow results.

To check the robustness and performance of the frequency partitioning algorithm based on graph theory for different operating conditions, different tests have been performed. Disturbances are applied to ramp up all system loads simultaneously. The disturbances have been initiated at 3\% and increased to $8 \%$. The partitions stay approximately the same with some minor changes near the boundaries. Figure 3 shows the result of partitioning considering 4 partitions.

\section{A. $3 \quad N C P N$ frequency graph partitioning considering $P V$ and Thermal Solar}

In the following section, impact of PV and Thermal Solar is considered in the partitioning of NCPN. 200 MW active power is added to the NCPN in ATACAMA area. Figure 4 shows the results of partitioning in 3 regions with this new reconfiguration of the system.

As can be seen from the above figures, adding PV and Thermal Solar to the NCPN will change the partitioning structure.

\section{DIAGRAMA UNILINEAL SIMPLIFICADO DEL SING}

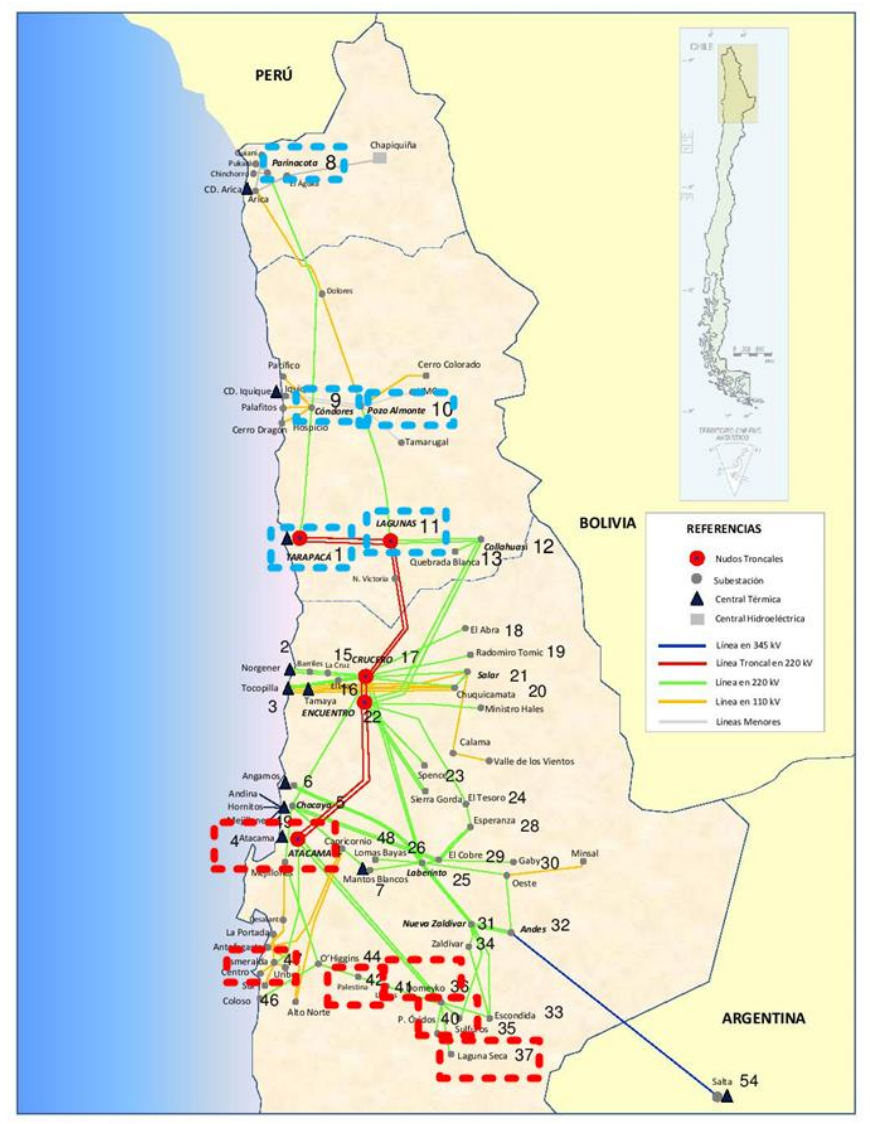

Fig. 2. Graph partitioning of NCPN considering three regions on the geographical map.

It can be seen that in the new partitioning, the buses Encuentro (22), Collahuasi (12), Quebrada Blanca (13), Spence (23) and El Tesoro (24) has been added to the partitioning where Atacama generator is there. The results show adding the PV and Thermal Solar can change the frequency partitioning and future control of the network.

\section{Voltage Partitioning Algorithm}

For voltage control, as figure 5 shows, voltage on buses of each partition will be measured and reactive power will be injected into partitions to compensate voltage variations. The importance of voltage partitioning is to obtain the required reactive power injected in each partition. A power plow in different situations considering different contingencies will be considered to verify the partitions.

\section{A.1 Weighted Graph model of the NCPN}




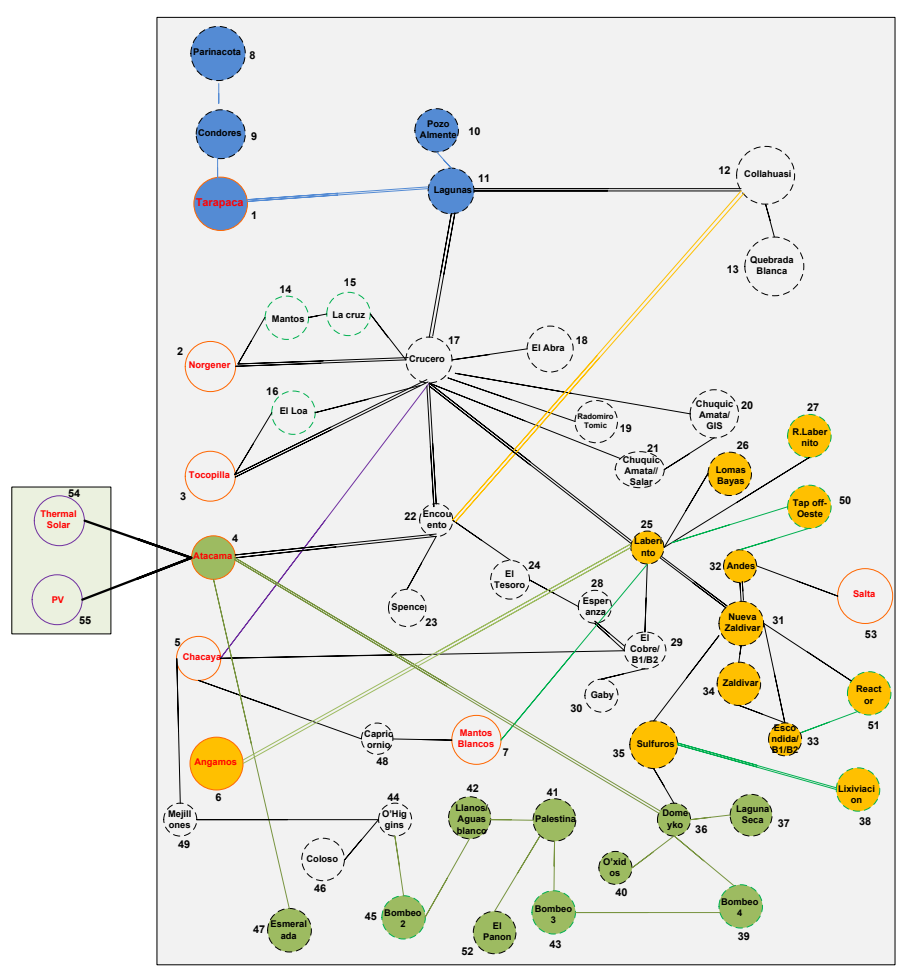

Fig. 3. Graph partitioning of NCPN considering four regions.

A power flow has been performed in normal situation of NCPN to find the buses voltage. This information will be used as weighting of graph connection matrix between buses. Buses voltage will be used for voltage partitioning technique.

\section{A. $2 \quad N C P N$ voltage graph partitioning}

The Graph Theory algorithm has been performed to find three partitions for NCPN. The results can be seen in the figure 6 . As can be seen in this figure, firstly, three partitions have been considered.

In this partitioning, PV and Thermal Solar are not connected to the NCPN. Buses are included in each partition has been differently colored. The buses voltage are considered as weights and partitioning has been performed based on power flow results. The weights on the transmission lines for the voltage partitioning are calculated as follows:

$$
=\sqrt{\left(v_{\text {normal }}\right)^{2}+\left(v_{\text {total_Dist }}\right)^{2}+\left(v_{\text {some_Dist }}\right)^{2}}
$$

where $\omega_{i j}$ is the weight on the transmission lines between buses $i$ and $j, v_{\text {normal }}$ is the voltage buses in the normal situation of the NCPN and is calculated as:

$$
v_{\text {normal }}=\sqrt{\left(v_{\text {bus } \_j}^{\text {normal }}-v_{\text {bus } \_i}^{\text {normal }}\right)^{2}}
$$

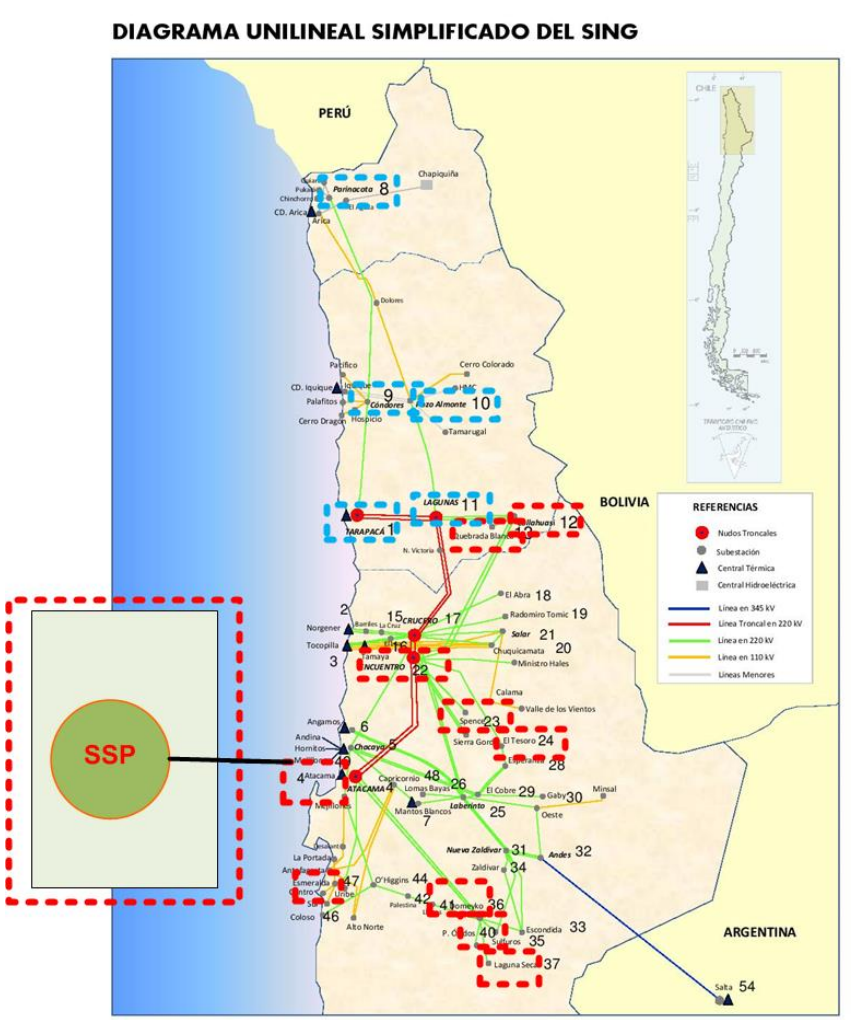

Fig. 4. Graph partitioning of NCPN considering three regions adding PV and Thermal Solar on the geographical map.

where $v_{b u s_{-} j}^{\text {normal }}$ is the voltage on the bus $j$ in the normal situation, $v_{\text {total_Dist }}$ is the voltage buses when disturbances are applied to the all buses of the NCPN and is calculated as:

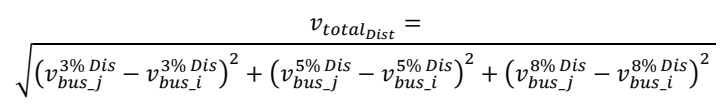

where $v_{\text {bus }}^{3 \%}$ Dis $i s$ the voltage on the bus $j$ after adding $3 \%$ disturbances on the all buses of the NCPN, $v_{\text {total_Dist }}$ is the voltage buses when disturbances are applied to some buses of the NCPN (these buses are randomly chosen) and is calculated as:

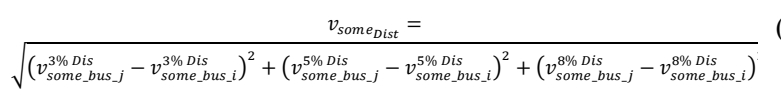

where $v_{\text {some_bus } j}^{3 \%}$ is the voltage on the bus $j$ after adding $3 \%$ disturbances on the some buses of the NCPN.

\section{A. $3 \quad N C P N$ voltage graph partitioning considering $P V$ and Thermal Solar}

Here, impact of PV and Thermal Solar is considered in the partitioning of NCPN. $200 \mathrm{MW}$ active power is added to the NCPN in ATACAMA generation. The results for voltage partitioning stays the same. The results of partitioning with new re-configuration of the system is not changing for voltage partitioning. 


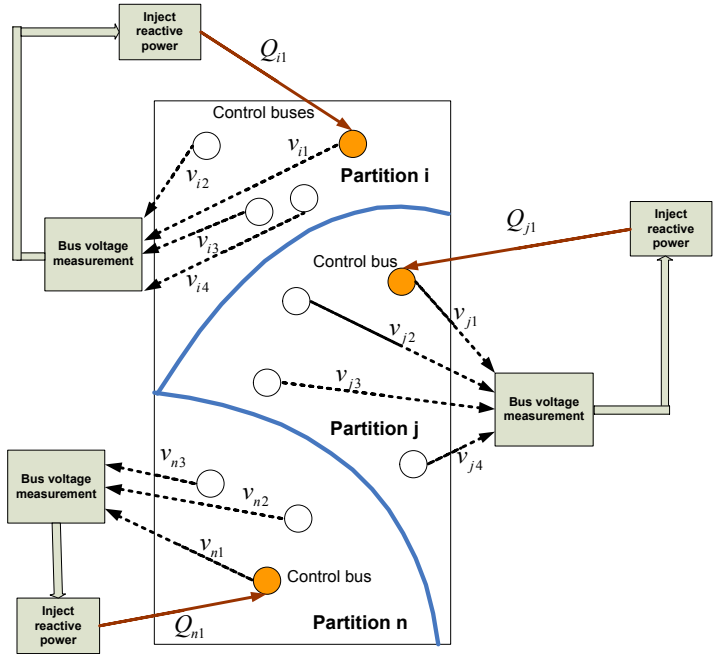

Fig. 5. Partitioning for voltage control.

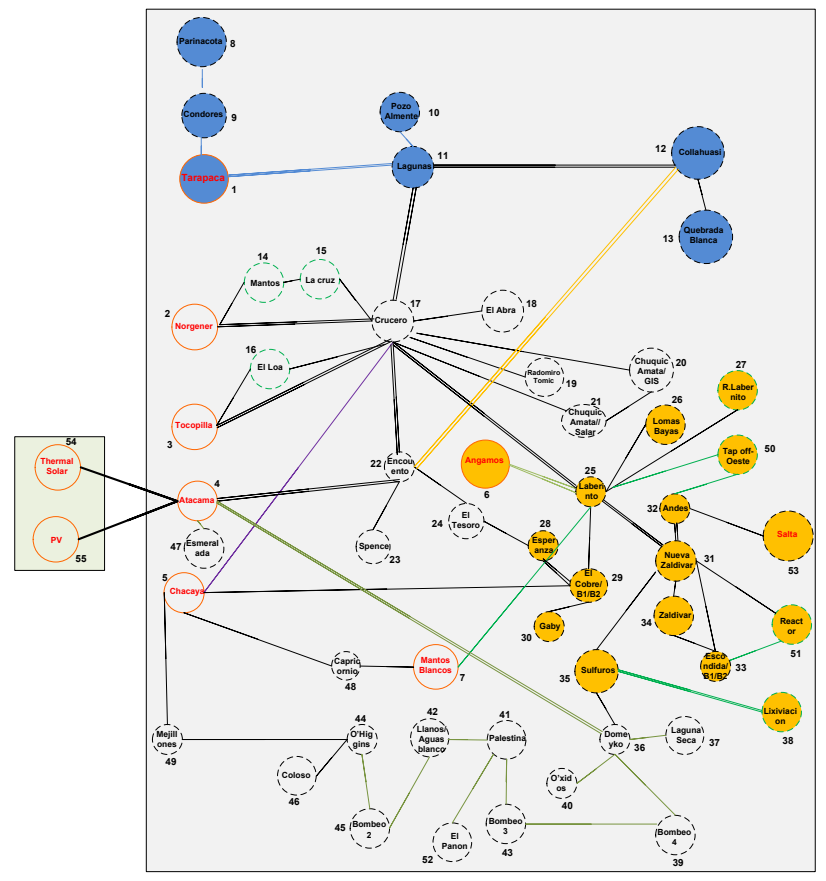

Fig. 6. Graph voltage partitioning of NCPN considering three regions.

\section{CONCLUSION}

Frequency and voltage control of power systems are essential as these systems operating closer to their limits. High penetration of renewable energy resources changes the infrastructure of the traditional power systems. There are a lot of advantages to perform control of power systems in a decentralized conception. The partitioning have been performed in this paper using graph theory gives good results to convert large scale power system to smaller ones to have better capability of control. Two different technique have been presented for frequency and voltage partitioning. The results have been applied to real network of Chile power system and shows the performance of the algorithm. The results have also performed in the case if renewable energy integration in Chile power network and shows how the partitioning change with effects of penetration of renewable energy sources.

\section{REFERENCES}

[1] A. Keyhani, and A. Chatterjee, "Automatic Generation Control Structure for Smart Power Grids," IEEE Transactions on Smart Grid, Vol. 3, no. 3, pp. 1310-1316, 2012.

[2] G. Delille, B. Francois, G. Malarange, "Dynamic Frequency Control Support by Energy Storage to Reduce the Impact of Wind and Solar Generation on Isolated Power System's Inertia," IEEE Transactions on Sustainable Energy, Vol. 3 , no. 4, pp. 931-939, 2012.

[3] M. Datta, T. Senjyu, A. Yona, T. Funabashi, and K. Chul-Hwan, "A Frequency-Control Approach by Photovoltaic Generator in a PV-Diesel Hybrid Power System," IEEE Transactions on Energy Conversion, Vol. 26 , no. 2, pp. 559 - $571,2011$.

[4] M. Jonsson, M. Begovic, and J. Daalder, "A new method suitable for real-time generator coherency determination," IEEE Transactions on Power Systems., vol. 19, no. 3, pp. 1473-1482, Aug. 2004.

[5] S. K. Joo, C. C. Liu, and L. E. Jones, "Coherency and aggregations techniques incorporating rotor and voltage dynamics," IEEE Transactions on Power Systems., vol. 19, no. 2, pp. 1068-1075, May 2004.

[6] S.-K. Joo, C.-C. Liu, L. E. Jones, and J.-W. Choe, "Coherency and aggregation techniques incorporating rotor and voltage dynamics," IEEE Trans. Power Syst., vol. 19, pp. 1068-1075, 2004.

[7] E. De Tuglie, S. M. Iannone, and F. Torelli, "A coherency recognition based on structural decomposition procedure," IEEE Trans. Power Syst., vol. 23, pp. 555-563, 2008.

[8] I. Kamwa, A. K. Pradhan, and G. Joos, "Automatic segmentation of large power systems into fuzzy coherent areas for dynamic vulnerability assessment," IEEE Trans. Power Syst., vol. 22, no. 4, pp.1974-1985, Nov. 2007.

[9] M. Jonsson, M. Begovic, and J. Daalder, "A new method suitable for real-time generator coherency determination," IEEE Trans. Power Syst., vol. 19, no. 3, pp. 1473-1482, Aug. 2004.

[10] E. D. Tuglie, S. M. Iannone, and F. Torelli, "A Coherency Recognition Based on Structural Decomposition Procedure," IEEE Transactions on Power Systems, vol. 23, no. 2, pp. 553-563, May. 2008.

[11] H. Mehrjerdi, E. Ghahremani, S Lefebvre, M. Saad, and D. Asber, "An Authenticated Voltage Control of Partitioned Power Networks with Optimal Allocation of STATCOM Using Heuristic Algorithm," IET Gener, Transm. Distrib, vol. 7, no.9, pp. 1037-1045, 2013.

[12] H. Mehrjerdi, S Lefebvre, M. Saad, and D. Asber, "A decentralized control of partitioned power networks for voltage regulation and prevention against disturbance propagation" IEEE Transactions on Power Systems., vol. 28, no. 2, pp. 1461-1469, 2013.

[13] J. Li, C. C. Liu, and K. P. Schneider, "Controlled Partitioning of a Power Network Considering Real and Reactive Power Balance," IEEE Transactions on Smart Grid, vol. 1, no. 3, pp. 261-269, Nov. 2010.

[14] I. Kamwa, A. K. Pradhan, G. Joos, and S. R. Samantaray, "Fuzzy Partitioning of a Real Power System for Dynamic Vulnerability Assessment," IEEE Transactions on Power Systems., vol. 24, no. 3, pp.1356-1365, Aug. 2009.

[15] A. Pothen, H. D. Simon, and K.-P. Liou, "Partitioning sparse matrices with eigenvectors of graphs," SIAM J. Matrix Anal. Appl., vol. 11, pp.430-452, 1990.

[16] A. Pothen, H. D. Simon, L. Wang, and S. T. Bernard, "Towards a fast implementation of spectral nested dissection, " in Supercomputing '92 Proceedings, IEEE Computer Society Press, Washington, DC, 1992, pp. 42-51.

[17] R. Ponnusamy, N. Mansour, A. Choudhary, and G. C. Fox, "Graph contraction and physical optimization methods: A quality-cost tradeo for mapping data on parallel computers," in International Conference of Supercomputing, ACM, New York, 1993.

[18] P. K. Chan, D. F. Schlag, and J.Y. Zien, "Spectral K-way Ratio-Cut Partitioning and Clustering," IEEE Transactions on Computer Aided Design of Integrated Circuits and Systems., vol. 11, no. 9, pp. 10881096, Sep 1994. 